\title{
Jiangzhi Ligan Decoction Alleviated Nonalcoholic Fatty Liver Disease Induced by High-Fat-Diet in Rats via GSDMD-mediated Canonical/Non-canonical Pyroptosis Pathway
}

\section{XIAO ZHOU}

Hunan University of Chinese Medicine https://orcid.org/0000-0002-0443-4151

Kangkang Yin

Hunan University of Chinese Medicine

Wei Jiang

Hunan University of Chinese Medicine

\section{Ziwei Dai}

Hunan University of Chinese Medicine

Biao Tang ( $\nabla$ biaotang@hnucm.edu.cn)

Hunan University of Chinese Medicine

\section{Research}

Keywords: Pyroptosis, Nonalcoholic fatty liver disease (NAFLD), Jiangzhi Ligan Decoction (JZLGD), Lipid metabolism disorder, Lipid accumulation, Inflammation

Posted Date: February 24th, 2021

DOI: https://doi.org/10.21203/rs.3.rs-250732/v1

License: (c) (i) This work is licensed under a Creative Commons Attribution 4.0 International License.

Read Full License 


\section{Abstract \\ Background}

Hepatoprotective effects of Chinese herbal formula Jiangzhi Ligan Decoction (JZLGD) against nonalcoholic fatty liver disease (NAFLD) have been demonstrated, but its mechanism is not clear. The aim of this study was to evaluate the protective effects of against high-fat diet (HFD) induced NAFLD in rat, and to further explore the potential molecular mechanism.

\section{Methods}

SD rats were assigned to five different groups: normal control group, NAFLD model group and JZLGDtreated NAFLD group (3 doses of JZLGD: $2.3,4.6,9.2 \mathrm{~g} / \mathrm{kg}$ of body weight, respectively). All the rats were fed a HFD for 18 weeks except the normal control group(a normal diet). After 12 weeks, rats in JZLGDtreated NAFLD group were administered different doses of JZLGD by oral gavage once daily for another period of 6 weeks, and the rest were given the same dosage of normal saline. After the intervention, blood and liver from each sample were carefully removed and analyzed accordingly.

\section{Results}

We found that JZLGD significantly reduced the liver index, levels of alanine aminotransferase (ALT) and aspartate aminotransferase (AST) in serum. Furthermore, pathological examinations showed that JZLGD markedly reduced liver lipid droplets and improved liver lipid accumulation, NAFLD activity score and ballooning pathology scoring were also decreased. The detection of cytokines showed that JZLGD could significantly reduced the levels of serum inflammatory factors IL-1 $\beta$, IL-18, tumor necrosis factor a (TNFa) and IL-6, protected HFD rats from inflammation. In addition, NAFLD treatment, exhibited significant reduction in serum triglyceride (TG), total cholesterol (TC), low density lipoprotein (LDL) and free fatty acid (FFA) when compared to NAFLD control rats. JZLGD intervention also reduced the level of serum lipopolysaccharide (LPS) and the expression of NOD-like receptor family pyrin domain containing 3 (NLRP3), apoptosis-associated speck-like protein containing a CARD (ASC), caspase-1, caspase-11, GSDMD, GSDMD N-terminus (GSDMD-N), IL-1 $1 \beta$, IL-18 in the liver.

\section{Conclusion}

These results demonstrate the hepatoprotective effects of JZLGD in NAFLD mice, the effects may be mediated via downregulation of NLRP3 / caspase-1/GSDMD mediated canonical pyroptosis pathway and LPS /caspase-11/GSDMD mediated non-canonical pyroptosis pathway.

\section{Background}


Non-alcoholic fatty liver disease (NAFLD) is a rapidly increasing global health problem especially in the United States, the prevalence of NAFLD is as high as $20 \%-30 \%[1]$, also the prevalence rate in the Asia is $7 \%-20 \%[2]$. Currently, with the changes in people's diet and lifestyle, the incidence of NAFLD has gradually become younger aggregating burden on public-health-care systems. Even worse, some patients will progress to hepatitis, liver fibrosis and cirrhosis, and eventually develop hepatocellular carcinoma, however, currently, there are no effective drugs for the treatment of NAFLD [3], so it is very significantly to research the therapy target for NAFLD.

The pathogenesis of NAFLD is related to lipid metabolism disorder, lipid accumulation, insulin resistance, and inflammation $[4,5]$. In particular, hepatic inflammation is an essential pathophysiological process during NAFLD that unites the lipotoxic response with the generation of oxidative stress [6]. Additionally, studies showed that inflammatory activation results in hepatic insulin resistance and steatohepatitis[7]. The occurrence and development of inflammation will aggravate the deterioration of NAFLD, and make NAFLD progress into cirrhosis. Pyroptosis is a form of pro-inflammatory cell death and gasdermin $D$ (GSDMD) is the executive molecule[8]. Cleavage of the GSDMD by caspase- 1 , and caspase- $4 / 5 / 11$ leads to translocation of the $\mathrm{N}$-terminal fragment to the plasma membrane, which then forms pores $(10-15 \mathrm{~nm}$ in diameter) that facilitate pyroptotic cell death to release interleukin-1 $\beta$ (IL-I $\beta$ ) and interleukin-18(IL-18) through the pore formed by GSDMD-N oligomerization[9, 10,11]. On the one hand, the activation of caspase-1 required NOD-like receptor family pyrin domain containing 3 (NLRP3) inflammasome which recognizes diverse stimuli including pathogen-associated molecular patterns(PAMPs) and endogenous danger signals(DAMPs) to activate pro-caspase-1 cleaves into form active caspase-1[12].This process of pyroptosis mediated by NLRP3/caspase-1/GSDMD is called as the canonical pyroptosis pathway. On the other hand, the occurrence of non-canonical inflammasome-mediated pyroptosis depended on caspase$11 / 4 / 5$ to recognize and bind lipopolysac-charides (LPS), then activates GSDMD to cause noncannonical pyroptosis pathway $[13,14,15]$. Recent studies have showed that pyroptosis which mediated the death of hepatocytes and aggravated the process of inflammation and fibrosis in the pathological development of NAFLD has become an important potential target of NAFLD treatment intervention[16].

In Chinese medicine philosophy, the pathogenesis of NAFLD includes spleen vacuity, liver stagnation, and phlegm-damp obstruction[17].Traditional Chinese medicine is effective in treating NAFLD, but there are few related studies. Jiangzhi Ligan Decoction (JZLGD), as a classical Chinese herbal formula, is a clinically experienced prescription and commonly used in clinical practice, which is composed of 6 kind of Chinese medicines including alisma orientalis (Zexie), salvia miltiorrhiza (Danshen), cassia seed (Juemingzi), turmeric (Yujin), seaweed (Haizao) and lotus leaf (Heye). Previous experiment showed JZLGD has a noticeably effect of regulating lipid metabolism, protecting liver and losing weight, and has been demonstrated playing an important role in treating NAFLD, but its mechanism is not clear. Pyroptosis is closely related to inflammatory response, and plays an indispensable role in the development of NAFLD. Inhibition of pyroptosis and inflammation in NAFLD model can protect liver from inflammation and delay the progression of the liver disease, hence, we hypothesized that JZLGD slow the progression of NAFLD may be related to the canonical and non-canonical inflammasome signaling 
pathways. Therefore, model of NAFLD rats were established to evaluate the improvement of JZLGD on NAFLD and further explored the potential molecular mechanism.

\section{Methods}

\section{Materials}

Reagent: Chloral hydrate (8MQ20-CC) was purchased from TCI Shanghai; Western and IP cell lysate (P0013) was purchased from Beyotime Biotechnology; Protease inhibitor cocktail (without EDTA, 100x DMSO storage solution) (B14002) and phosphatase inhibitor cocktail $(100 \times$ B15002) were purchased from Bimake; Rat IL-1 $\beta$ ELISA Kit (GN-R30172), rat IL-18 ELISA Kit (GN-R30168), rat TNF-a ELISA Kit (GNR31092) and rat IL-6 ELISA Kit (GN-R30201) were purchased from Gaining Biological; LPS detection kit (H178) was purchased from NanJing JianCheng Bioengineering Institute; GSDMD mouse monoclonal antibody (SC-393581) and caspase-11 (SC-374615) were purchased from Santa Cruz Biotechnology; GSDMD-N rabbit monoclonal antibody (93709) was purchased from Cell Signaling Technology; Caspase1 (NBP1-45433), NLRP3 (NBP2-12446) Rabbit anti polyclonal antibodies were purchased from Novus Biologicals; ASC (EPR10402(B))Rabbit monoclonal antibody was purchased from abcam; $\beta$-actin mouse anti monoclonal antibody (A5316) was purchased from Sigma; Goat anti rabbit second antibody (AP132P) and Goat anti mouse second antibody (AP124P) were purchased from Merck.

Instrument: Microplate Reader, Electrophoresis apparatus, Film transfer apparatus and Gel imaging system (BIO-RAD, USA), LEICADNLB2 binocular microscope (LEICA company), Shandon325 paraffin slicing machine (British Shandon company), Fall-automatic biochemical analyzer.

\section{Preparation of Jiangzhi Ligan Decoction}

JZLGD is composed of Alisma orientalis(Zexie) $10 \mathrm{~g}$, Cassia seed(Juemingzi)30 g, Salvia miltiorrhiza(Danshen) $10 \mathrm{~g}$, Turmeric (Yujin) $10 \mathrm{~g}$, Seaweed(Haizao) $30 \mathrm{~g}$ and Lotus leaf (Heye)10 g. All the Chinese herbal pieces were purchased from The First Hospital of Hunan University of Chinese Medicine. The above drugs were soaked in 8 times of water for $30 \mathrm{~min}$, boiled for $1 \mathrm{~h}$, then filtered, added 6 times of water, boiled for $1 \mathrm{~h}$, and then filtered. We mixed the liquid medicine and concentrated it to $2 \mathrm{~g}$ $/ \mathrm{ml}$ by using Rotary Evaporator at $65^{\circ} \mathrm{C}$, and stored it at $4{ }^{\circ} \mathrm{C}$. Before use, it was diluted to the required concentration with normal saline.

\section{Animals and treatment}

Sprague-Dawley(SD) male rats $(150 \mathrm{~g} \pm 10 \mathrm{~g})$ were purchased from Hunan SJA Laboratory Animal Co.هLtd and acclimatized for 7 days. Rats were kept in controlled ambient temperature $\left(24 \pm 2{ }^{\circ} \mathrm{C}\right)$ and humidity (60 $\pm 10 \%)$ under $12 \mathrm{~h}$ light-dark cycles; water was available ad libitum. This study was reviewed and approved by the Animal Experiment Ethics Committee of Hunan University of Chinese Medicine and carried out in accordance with their recommendations. 
According to the previous study, the rats received a high-fat diet (HFD) (40 kcal\% fat, $20 \mathrm{kcal} \%$ sucrose, and $2 \%$ cholesterol) for 12 weeks to develop NAFLD. The high-fat diet was provided by

SD rats were randomly divided into five groups: normal control group, NAFLD model group and JZLGDtreated NAFLD group (3 doses of JZLGD: $2.3,4.6,9.2 \mathrm{~g} / \mathrm{kg}$ of body weight, respectively). The normal control group were fed a normal $\operatorname{diet}(\mathrm{ND})$, while the rest of the groups received a HFD during the experimentation period (12 weeks +6 weeks). After 12 weeks, rats in JZLGD-treated NAFLD group were administered different doses of JZLGD by oral gavage once daily for another period of 6 weeks, and the rest were given the same dosage of normal saline. Food intake was monitored daily and body weight was measured weekly during the experiment. After the intervention, blood and liver from each sample were rapidly removed and stored at $-80^{\circ} \mathrm{C}$ for further studies.

\section{Liver index}

After 18 weeks, the rats were anesthetized by injecting with $1 \%$ pentobarbital sodium after overnight fasting. The body weight and liver weight were measured, and the following formula was used for determining the liver index: liver index $=($ liver weight/body weight $) * 100 \%$.

\section{Histopathology analysis}

For histopathological analysis, we took the same segments of liver tissue from each group and washed it with ice-cold saline for 1-2 times, then kept them in $4 \%$ paraformaldehyde. $4 \mu \mathrm{m}$ thickness tissue were embedded in paraffin blocks and stained with hematoxylin-eosin. Three practiced pathologists who were blinded to the study design performed all the histopathological examination, then scored the ballooning degeneration score and the NAFLD activity score. The scoring criteria were in accordance with the guidelines of the National Institutes of health clinical research network on non-alcoholic steatohepatitis.

\section{Biochemical estimations}

The blood samples were centrifuged at $3000 \mathrm{r} / \mathrm{min}$ for $10 \mathrm{~min}$ to separate the serum. We used fallautomatic biochemical analyzer to evaluate serum activity of liver enzymes (alanine transaminase enzyme (ALT) and aspartate transaminase enzyme (AST)) and serum level of lipid profile (triglyceride(TG), total cholesterol(TC), low density lipoprotein(LDL) and free fatty acids(FFA)).

\section{Assessment of serum IL-1 $\beta$, L-18, TNF- $a$, IL-6}

Blood was obtained from abdominal aorta and centrifuged to get the serum. The levels of IL-1 $\beta, L-18$, tumor necrosis factor $\mathrm{a}(\mathrm{TNF}-\mathrm{a})$ and IL- 6 in serum were determined by enzyme-linked immunosorbent assay technique (ELISA) according to standard instructions.

\section{The levels of serum LPS}

Blood was obtained from the abdominal vein and placed at room temperature for 30 minutes, then centrifuged to separate the serum. The level of serum LPS was measured with a kit according to the 
manufacturer's instructions. Firstly, samples were added into the enzyme labeled wells which had been coated with antibodies, and then labeled recognition antigens were added. The mixture was allowed to incubate at room temperature for $1 \mathrm{~h}$. Afterwards, the plate was washed with PBST three times, then we added avid in HRP and made them incubated at $37^{\circ} \mathrm{C}$ for $1 \mathrm{~h}$. The absorbance was measured at $450 \mathrm{~nm}$. The standard curve was drawn according to the absorbance of standard pore and its corresponding concentration, and the concentration of LPS in serum of each group could be calculated.

\section{Western blot analysis}

We randomly selected five mice in each group, and took the same segments of liver tissue $100 \mathrm{mg}$. Then $1 \mathrm{ml}$ RIPA (with cocktail and phosphatase inhibitor added) was added. After homogenize on ice with a glass homogenizer for $30 \mathrm{~min}$, the homogenate was centrifuged to obtain the hepatic tissue proteins, the concentration of protein extracted was determined with BCA. We took 40 ug protein in each group for the follow-up experiment. Protein samples were separated by SDS-PAGE gels and transferred to PVDF membranes. After blocking with $5 \%$ nonfat milk in TBST for $1 \mathrm{~h}$, membranes were incubated at $4{ }^{\circ} \mathrm{C}$ overnight with the primary antibodies against NLRP3 (1:1000), apoptosis-associated speck-like protein containing a CARD(ASC)(1:1500), Caspase-1 (1:500), Caspase-11 (1:500), GSDMD (1:1000), GSDMD Nterminus(GSDMD-N) (1:1000), IL-1 $\beta$ (1:1000), IL-18 (1:1000) and $\beta$-actin (1:5000). After washing the membrane with TBST, the membranes were incubated at room temperature with secondary antibodies for $1 \mathrm{~h}$. The protein bands were visualized with Western Bright ECL. Finally, Quantity One software was used for the quantitative grayscale analysis of the band

\section{Statistical analysis}

The grouped data were analyzed with SPSS 22.0. Comparison of two groups was performed by two tailed Student's t-test. Comparison among three or more groups were analyzed with one-way ANOVA test. Results were expressed as the mean $\pm \mathrm{SD}$ (standard deviation). The GraphPad Prism6.0 software was used for statistical evaluations. $P<0.05$ was considered to indicate a statistically significant difference.

\section{Results}

\section{JZLGD Reduced Liver Index, Serum Lipid Levels, and Protects Injured Liver}

To evaluate the therapeutic effect of JZLGD on NAFLD in the HFD-fed rat model, we detected the body weight, liver weight, liver index, serum lipid levels and liver function parameters. Compared to the control group, the body and liver weight and liver index in NAFLD model group apparently increased. JZLGD treatment for 6 weeks significantly decreased body weight, liver weight and liver index. As shown in figure $1(d)(e)(f)(g)$ compared to HFD group, serum TG, TC, FFA, LDL levels were noticeably decreased after JZLGD treatment. The progression of NAFLD significantly induced the higher levels of both liver injuryrelated markers ALT and AST compared with the control rats. Corresponding, JZLGD showed significantly lower serum circulating ALT and AST levels, which indicated that JZLGD can protect rats from HFD induced liver injury. 


\section{JZLGD Alleviated Pathological Morphology of Liver Tissue and Inflammation}

To further confirm the effect of JZLGD on the liver histopathology of NAFLD rats, we performed H\&E staining, NAFLD activity score(NAS) and ballooning score. Compared with the control group, the livers from rats on a high fat diet showed typical fat deposition and hepatic steatosis, accompanied by hepatocyte ballooning and lobular inflammation. These distinguishing features implied that HFD feeding can induce NAFLD and developed hepatic steatosis. With the intervention of JZLGD, hepatic steatosis and lipid accumulation gradually decreased, at the same time, the NAFLD model group ballooning score and NAS were significantly increased, while the intervention of JZLGD significantly reduced the ballooning score and NAS. The extent of its improvement was concentration-dependent.

Then we detected the related inflammatory factors to assess the degree of hepatic inflammation in mice fed with high-fat diet, which showed there were higher IL-1 $1 \beta$, IL-18, TNF-a and IL-6 levels in serum of the HFD induced rats relative to the normal group. Similarly, in mice fed with JZLGD, IL-1 $\beta$, IL-18, TNF-a and IL-6 were decreased obviously relative to HFD fed mice alone.

\section{JZLGD Inhibited the Expression of GSDMD and GSDMD-N}

We used western boltting to detected the key protein GSDMD which is the ultimate executor and the most critical part of cell pyroptosis, besides, its active form GSDMD-N in liver tissues also were detected to further research its related mechanism. From figure 3, it was found that GSDMD and GSDMD-N were obviously over-expressed in livers in the NAFLD model group compared with the control group; GSDMD and GSDMD-N levels in livers were significantly decreased after treatment with JZLGD.

\section{JZLGD Suppressed the Levels of NLRP3, ASC, caspase-1, IL-1ß, IL-18}

To further explore the effect of JZLGD on GSDMD-mediated canonical pyroptosis pathway, we detected NLRP3, caspase-1, and their adaptor protein ASC. Compared with the normal group, the expression levels of NLRP3, caspase-1, and ASC protein in liver tissue of model group were significantly increased. However, with the treatment of JZLGD, NLRP3, caspase-1, and ASC protein were significantly decreased. Moreover, IL-1 $\beta$, IL-18 in liver tissue were detected, the result showed that the IL-1 $1 \beta$, IL-18 levels of rats feeding with HFD were higher than normal diet rats, and JZLGD suppressed the expression of IL-1ß, IL-18 compared with HFD rats.

\section{JZLGD Suppressed the expression of caspase-11 and LPS}

Compared with the normal group, the expression of caspase-11 protein in the liver tissue of the model group was significantly increased; compared with the model group, the expression of caspase-11 protein in the liver tissue of the JZLGD group was significantly reduced; At the same time, HFD markedly increased serum LPS concentrations. Treatment with JZLGD significantly decreased the LPS level elevation induced by HFD. 


\section{Discussion}

NAFLD is rapidly developing into the main cause of liver cancer and liver cirrhosis in the world, yet there is no approved treatment drug[18]. Contrarily, traditional Chinese medicine has the characteristics of a multi-ingredient and multipath pharmacological action, and been well-documented for its therapeutic effects for treatment of NAFLD $[19,20]$. JZLGD is a clinical experience prescription and has a history of clinical use for treatment of NAFLD, in this present study, the potential mechanism of JZLGD on NAFLD was explored firstly via the high fat diet induced NAFLD rats.

12 weeks of HFD feeding increased body weight, liver weight and liver index of HFD mice, however, after JZLGD administration, body weight, liver weight and liver index of NAFLD rats induced by high-fat diet were obviously reduced. Besides, lipid profile detection showed that JZLGD had the ability of regulating blood lipid levels. The activity of hepatic injury marker, such as ALT and AST, also tended to be lower in the JZLGD treated rats suggesting its role in prevention and alleviation of NAFLD in HFD fed rats. Subsequently, pathological examinations showed that JZLGD markedly mitigated liver lipid droplets and hepatic steatosis. Furthermore, NAFLD activity score and balloning score were decreased in HFD rats suggesting JZLGD slowing down lipid accumulation and steatosis. Hence, all these findings illustrated that JZLGD indeed had a good therapeutic effect for NAFLD.

In sequencing studies, we observed that high fat diets markedly increased hepatic inflammatory factors such as IL-1 $\beta$, IL-18, TNF- $\alpha$ and IL-6. Treatment with JZLGD obviously decreased these high levels compared with the NAFLD model group, which suggested JZLGD attenuated hepatocelluar inflammation. As the core of the pathogenesis of NAFLD, inflammatory response plays an important role in the occurrence and development of NAFLD $[21,22]$. Therefore, we speculated the protective effect of JZLGD against NAFLD occured probably through anti-inflammatory response. Recent studies have shown that pyroptosis, as an inflammation-related and controllable cell death method with potential therapeutic implications of NAFLD[23], accelerates the process of hepatic inflammation and fibrosis[24, 25]. Noticeably, pyroptosis is the indispensable pathogenesis in the development of NAFLD model[23, 26], more importantly, suppression of inflammasome-dependent GSDMD-mediated cell pyroptosis could attenuate hepatic injury in liver diseases[27]. Therefore, we focused on the influence of JZLGD on pyroptosis in this study. So,we detected the major executor protein GSDMD firstly, which is a generic substrate of inflammatory caspases. GSDMD acts as a pyroptosis executor via its cleaved form GSDMD$\mathrm{N}$ that triggers pyroptosis and aggravates the released of pro-inflammatory factors like IL-1 $\beta$, IL-18 through the GSDMD-induced pores[24], apart from this, inhibiting the activation of GSDMD blocks the progression of NAFLD, hence, GSDMD is considered as a potential target for the treatment of NAFLD $[9$, 28]. Here, our experimental dates showed that the expression of GSDMD protein and its activated form GSDMD-N were significantly increased in NAFLD rats induced by high-fat diet, this is consistent with previous studies[9], which further verified that GSDMD plays an important role in the occurrence and development of NAFLD. However, the intervention of JZLGD reversed the high expression of GSDMD and GSDMD-N induced by HFD indicating that JZLGD could regulate the activation of GSDMD, so as to therapy NAFLD. 
GSDMD protein were cleaved to generate GSDMD-N depended on pro-inflammatory caspases-1, and caspase-4/5/11 via the canonical and non-canonical inflammasome signaling pathways[29]. Caspase-1 is the initiating factor of the canonical pyroptosis pathway and its activation required the activated of NLRP3[30]. Otherwise, caspase-1 directly cleaves pro-IL-1 $\beta$ and pro-IL-18 to generate the mature cytokines and controls their secretion to induce pyroptosis[31]. Our results had showed that, in addition to elevated GSDMD, NLRP3, ASC, caspase-1, IL-1 $\beta$ and IL-18 were also elevated in HFD-induced NAFLD mice, which suggested that there is also activation of inflammasome NLRP3 in NAFLD. The expression levels of NLRP3, caspase-1, IL-1 $\beta$ and IL-18 are key indicators for evaluating the activation of NLRP3 inflammasomes[32, 33]. The NLRP3 inflammasome transmits the recognition signal to ASC, which as the intermediate adaptor protein for signal amplification enables to activate caspase-1 in a large amount[34]. Then, caspase- 1 is subjected to stimulate directly pro-IL-1 $\beta / 18[35]$ and to cleave the key effector molecule of pyroptosis-GSDMD[36], both of which results in the massive release of IL-1 $\beta / 18$. In our present study, JZLGD treatment decreased NLRP3, ASC, caspase-1, IL-1 $\beta$ and IL-18 levels, suggesting that the treatment mechanism of JZLGD on high-fat-induced NAFLD mice may be related to the NLRP3-caspase-1-GSDMDmediated pyroptosis pathway.

Besides, in the non-canonical pyroptosis signaling, GSDMD is cleaved by caspase- 11 , and caspase- 11 is directly activated by LPS[37, 38]. The activated caspase-11 directly induces pyroptosis via the rupture of GSDMD-induced pores and subsequent membrane to release the pro-inflammatory factors[39]. The results showed that the cleaved forms of caspase-11 in liver tissue of NAFLD rats induced by high-fat diet was significantly increased, while in the JZLGD treatment group, we observed a significance decrease on caspase-11, implying that JZLGD may regulate the activation of GSDMD protein through inhibiting the express of caspase-11. There is evidence showing that the disorder of intestinal flora and the damage of intestinal mucosa can lead to the dissolution of gram-negative bacilli in the intestinal tract, resulting in the release of LPS into the portal vein blood, aggravating the liver inflammation and accelerating the disease progression of NAFLD $[40,41]$. Here, our data showed that the content of LPS in hepatic portal vein serum of NAFLD rats was significantly increased, and the intervention of JZLGD could significantly improve it. Based on the above results, we speculate that JZLGD may regulate the levels of LPS in serum of NAFLD rats to prevent the activation of caspase-11 and GSDMD, then reduce the release of proinflammatory factors and attenuate the process of NAFLD. Our findings illustrated JZLGD treatment prevented steatosis, inflammation, pyroptosis in rats fed with high fat, which may be related to LPScaspase-11-GSDMD mediated pyroptosis pathway.

\section{Conclusions}

In conclusion, all these findings demonstrated that there was a canonical /non- canonical activation of pyroptosis pathway in NAFLD rats induced by high-fat diet, while JZLGD prevented the activation of pyroptosis pathway by suppressed the expression level of GSDMD protein, and these results provided a new basis for the clinical treatment of NAFLD with JZLGD. In recent years, some studies have shown that GSDMD, as the executor of pyroptosis, is considered as a potential drug target for NAFLD treatment. Our study showed that JZLGD could regulate GSDMD mediated canonical / non-cannonical pyroptosis 
pathway to treatment rates fed with HFD, this is consistent with the current studies on NAFLD model, and suggested that GSDMD is a potential target for the treatment of NAFLD, but further verification is needed.

\section{Abbreviations}

JZLGD: Jiangzhi Ligan Decoction; NAFLD: nonalcoholic fatty liver disease; HFD: t high-fat diet; ALT: alanine aminotransferase; AST: aspartate aminotransferase; TNF-a: tumor necrosis factor a; TG: triglyceride; TC: total cholesterol; LDL: low density lipoprotein; FFA: free fatty acid; LPS: lipopolysaccharide; NLRP3: NOD-like receptor family pyrin domain containing 3; ASC: apoptosisassociated speck-like protein containing a CARD; GSDMD: caspases cleave gasdermin D

\section{Declarations}

\section{Ethics approval and consent to participate}

All experiments were reviewed and approved by the Animal Experiment Ethics Committee of Hunan University of Chinese Medicine and carried out in accordance with their recommendations.

\section{Consent for publication}

All authors consent to publish the present results.

\section{Availability of data and materials}

The data generated during this study are included in this article and results as shown in figures. And all data and materials will be available if needed.

\section{Competing interests}

The authors declare that there are no conflicts of interest.

\section{Funding Statement}

This work was supported by the Scientific Research Fund of Hunan Provincial Education Department (No. 18B244, 18B252)

\section{Authors' contributions}

Tang B designed this research and critically revised the manuscript. Yin-KK and Dai-ZW performed the experiments and carried out the biochemical analyses. Zhou $\mathrm{X}$ and Jiang $\mathrm{W}$ conducted the experiment, analyzed the experimental data and drafted the manuscript. All authors read and approved the final manuscript.

\section{References}


1. Bellentani S, Scaglioni F, Marino M, et al. Epidemiology of non-alcoholic fatty liver disease. Digestive diseases. 2010;28:155-61.

2. Wong V W S, Chan W K, Chitturi S, et al. Asia-Pacific Working Party on Non-alcoholic Fatty Liver Disease guidelines 2017-part 1: definition, risk factors and assessment. Journal of gastroenterology and hepatology. 2018;33:70-85.

3. Sanyal A J, Friedman S L, McCullough A J, et al. Challenges and opportunities in drug and biomarker development for nonalcoholic steatohepatitis: findings and recommendations from an American Association for the Study of Liver Diseases-US Food and Drug Administration Joint Workshop. Hepatology. 2015;61:1392-405.

4. de Alwis N M W, Day C P. Non-alcoholic fatty liver disease: the mist gradually clears. Journal of hepatology. 2008;48:104-12.

5. Braunersreuther V, Viviani G L, Mach F, et al. Role of cytokines and chemokines in non-alcoholic fatty liver disease. World journal of gastroenterology: WJG. 2012;18:727.

6. Yoon H, Cha B S. Pathogenesis and therapeutic approaches for non-alcoholic fatty liver disease. World journal of hepatology. 2014;6:800.

7. Tilg H, Moschen A R. Insulin resistance, inflammation, and non-alcoholic fatty liver disease. Trends in Endocrinology \& Metabolism. 2008;19:371-379.

8. Yang F, Zhu W, Cai X, et al. Minocycline alleviates NLRP3 inflammasome-dependent pyroptosis in monosodium glutamate-induced depressive rats. Biochemical and biophysical research communications. 2020;526:553-59.

9. Xu B, Jiang M, Chu Y, et al. Gasdermin D plays a key role as a pyroptosis executor of non-alcoholic steatohepatitis in humans and mice. Journal of hepatology. 2018;68:773-82.

10. Gaidt M M, Hornung V. Pore formation by GSDMD is the effector mechanism of pyroptosis. The EMBO journal. 2016;35:2167-169.

11. Silveira T N, Zamboni D S. Pore formation triggered by Legionella spp. is an Nlrc4 inflammasomedependent host cell response that precedes pyroptosis. Infection and immunity. 2010;78:1403-413.

12. Sborgi L, Rühl S, Mulvihill E, et al. GSDMD membrane pore formation constitutes the mechanism of pyroptotic cell death. The EMBO journal. 2016;35:1766-778.

13. Gan J, Huang M, Lan G, et al. High Glucose Induces the Loss of Retinal Pericytes Partly via NLRP3Caspase-1-GSDMD-Mediated Pyroptosis. BioMed Research International. 2020.

14. Jorgensen I, Miao E A. Pyroptotic cell death defends against intracellular pathogens. Immunological reviews. 2015;265:130-42.

15. Kuang S, Zheng J, Yang H, et al. Structure insight of GSDMD reveals the basis of GSDMD autoinhibition in cell pyroptosis. Proceedings of the National Academy of Sciences. 2017;114: 10642-647.

16. Al Mamun A, Wu Y, Jia C, et al. Role of pyroptosis in liver diseases. International immunopharmacology. 2020; 84:106489. 
17. Feng Y, Chen Y, Yang B, et al. Hepatoprotective Effect of Jianpi Huoxue Formula on Nonalcoholic Fatty Liver Disease Induced by Methionine-Choline-Deficient Diet in Rat. BioMed research international. 2019;doi:10.1155/2019/7465272

18. Paik J M, Golabi P, Younossi Y, et al. Changes in the global burden of chronic liver diseases from 2012 to 2017: the growing impact of NAFLD. Hepatology. 2020;72:1605-16.

19. Shi T, Wu L, Ma W, et al. Nonalcoholic fatty liver disease: pathogenesis and treatment in traditional Chinese medicine and Western medicine. Evidence-Based Complementary and Alternative Medicine. 2020;doi:10.1155/2020/8749564.

20. Yao H, Qiao Y J, Zhao Y L, et al. Herbal medicines and nonalcoholic fatty liver disease. World Journal of Gastroenterology. 2016; 22:6890.

21. Alisi A, Carpino G, Oliveira F L, et al. The role of tissue macrophage-mediated inflammation on NAFLD pathogenesis and its clinical implications. Mediators of inflammation. 2017;

22. Del Campo J A, Gallego P, Grande L. Role of inflammatory response in liver diseases: Therapeutic strategies. World journal of hepatology. 2018;10:1.

23. Guo H, Xie M, Zhou C, et al. The relevance of pyroptosis in the pathogenesis of liver diseases. Life sciences. 2019;223:69-73.

24. Beier J I, Banales J M. Pyroptosis: An inflammatory link between NAFLD and NASH with potential therapeutic implications. Journal of hepatology. 2018; 68:643-45.

25. Wu J, Lin S, Wan B, et al. Pyroptosis in liver disease: new insights into disease mechanisms. Aging and disease. 2019;10:1094.

26. Wree A, Eguchi A, McGeough M D, et al. NLRP3 inflammasome activation results in hepatocyte pyroptosis, liver inflammation, and fibrosis in mice. Hepatology. 2014;59:898-910.

27. Shi H, Zhang Y, Xing J, et al. Baicalin attenuates hepatic injury in non-alcoholic steatohepatitis cell model by suppressing inflammasome-dependent GSDMD-mediated cell pyroptosis. International immunopharmacology. 2020;81:106195.

28. Yi Y, Zheng J, Zhou Y, et al. Role of pyroptotic cell death in the pathogenesis of NASH. Biocell. 2020;44:7.

29. Drummer IV C E. Canonical and Non-canonical Inflammasomes are Differentially Regulated in Nafld, Which May Play Important Roles in Non-canonical Inflammasome Signaling in the Pathogenesis of Nonalcoholic Fatty Liver Disease and Vascular Inflammation. Arteriosclerosis, Thrombosis, and Vascular Biology. 2019;39 Suppl 1 : A298.

30. Willingham S B, Allen I C, Bergstralh D T, et al. NLRP3 (NALP3, Cryopyrin) facilitates in vivo caspase-1 activation, necrosis, and HMGB1 release via inflammasome-dependent and-independent pathways. The Journal of Immunology. 2009;183:2008-15.

31. Schneider K S, Groß C J, Dreier R F, et al. The inflammasome drives GSDMD-independent secondary pyroptosis and IL-1 release in the absence of caspase-1 protease activity. Cell reports. 2017;21:384659. 
32. Jha S, Srivastava S Y, Brickey W J, et al. The inflammasome sensor, NLRP3, regulates CNS inflammation and demyelination via caspase-1 and interleukin-18. Journal of Neuroscience. 2010; 30:15811-20.

33. Bauer C, Duewell P, Mayer C, et al. Colitis induced in mice with dextran sulfate sodium (DSS) is mediated by the NLRP3 inflammasome. Gut. 2010;59:1192-99.

34. Zhu W, Feng P P, He K, et al. Liraglutide protects non-alcoholic fatty liver disease via inhibiting NLRP3 inflammasome activation in a mouse model induced by high-fat diet. Biochemical and biophysical research communications. 2018;505:523-29.

35. Miao E A, Rajan J V, Aderem A. Caspase-1-induced pyroptotic cell death. Immunological reviews. 2011;243:206-14.

36. Shi J, Zhao Y, Wang K, et al. Cleavage of GSDMD by inflammatory caspases determines pyroptotic cell death. Nature. 2015;526:660-65.

37. Shi J, Zhao Y, Wang Y, et al. Inflammatory caspases are innate immune receptors for intracellular LPS. Nature. 2014;514:187-92.

38. Feng S, Fox D, Man S M. Mechanisms of gasdermin family members in inflammasome signaling and cell death. Journal of molecular biology. 2018;430:3068-80.

39. Evavold C L, Ruan J, Tan Y, et al. The pore-forming protein gasdermin D regulates interleukin-1 secretion from living macrophages. Immunity. 2018;48:35-44.

40. Maciejewska D, Łukomska A, Dec K, et al. Diet-Induced rat model of gradual development of nonalcoholic fatty liver disease (NAFLD) with lipopolysaccharides (LPS) secretion. Diagnostics. 2019;9: 205.

41. Leung $\mathrm{C}$, Rivera $\mathrm{L}$, Furness $\mathrm{J}$ B, et al. The role of the gut microbiota in NAFLD. Nature Reviews Gastroenterology \& Hepatology. 2016;13:412-25.

\section{Figures}



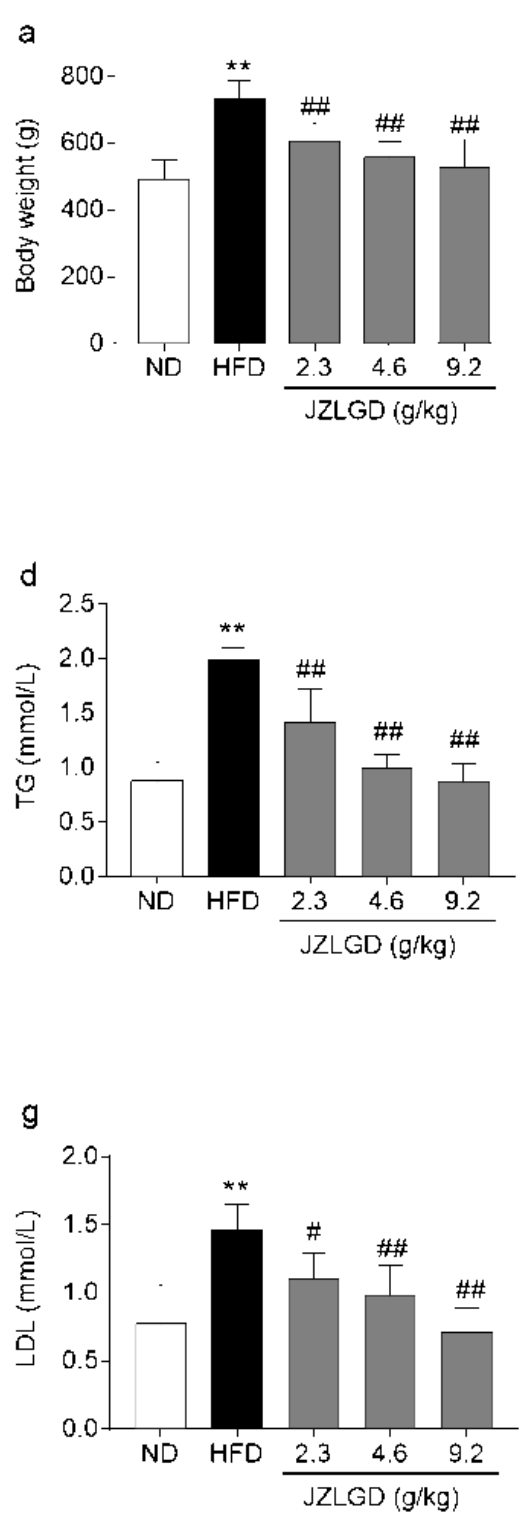

b

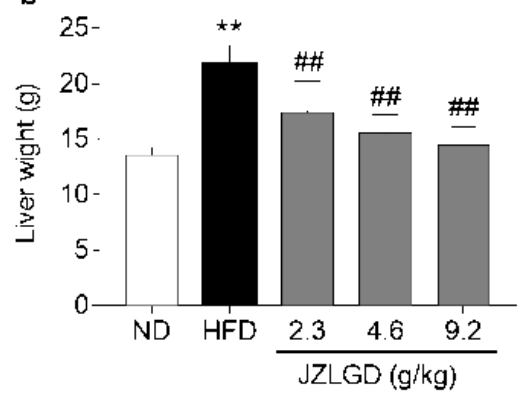

$\mathrm{e}$

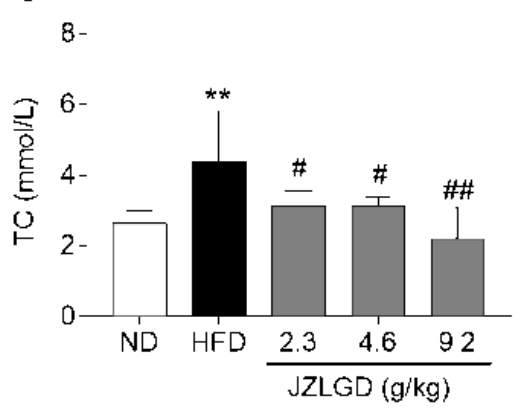

h

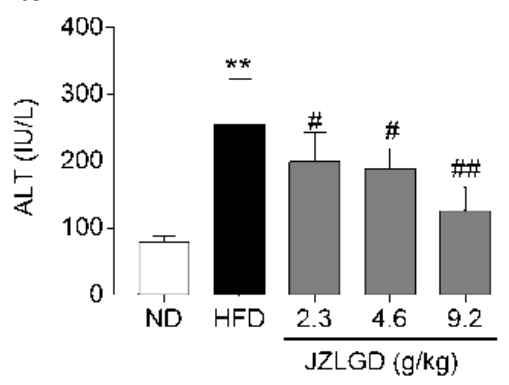

C

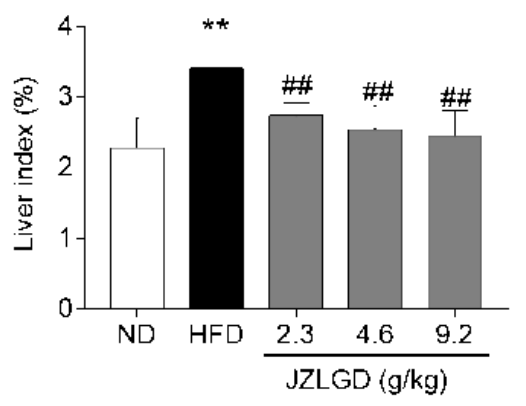

f

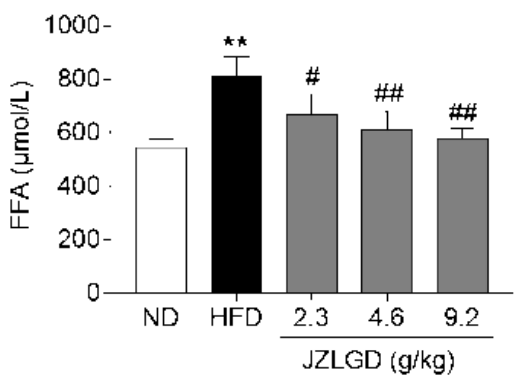

i

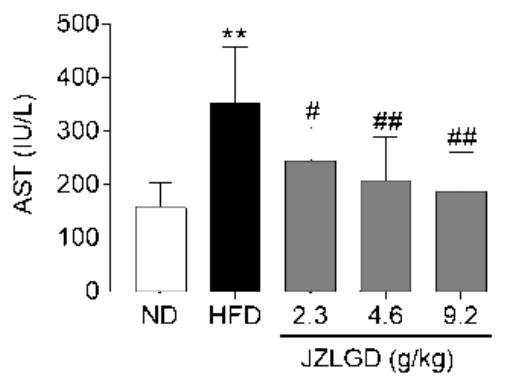

Figure 1

Effects of JZLGD on NAFLD rats and biochemical estimations. a Body weight of rats in different groups; b Liver weight of rats in different groups; c Liver index of rats in different groups; d Serum level of triglyceride(TG); e Serum level of total cholesterol(TC); f Serum level of free fatty acids(FFA); g Serum level of low density lipoprotein(LDL); $h$ Serum activity of liver enzymes alanine transaminase enzyme (ALT); i Serum activity of liver enzymes aspartate transaminase enzyme (AST). Data are expressed as the mean $\pm S D(n=10) ; * \star p<0.01$ vs. control; $\# p<0.05$, \#\#p<0.01 vs. model group. JZLGD, Jiangzhi Ligan Decoction; NAFLD, nonalcoholic fatty liver disease; SD, standard deviation 


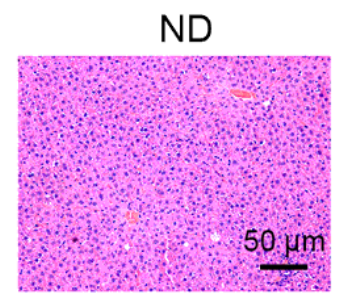

b

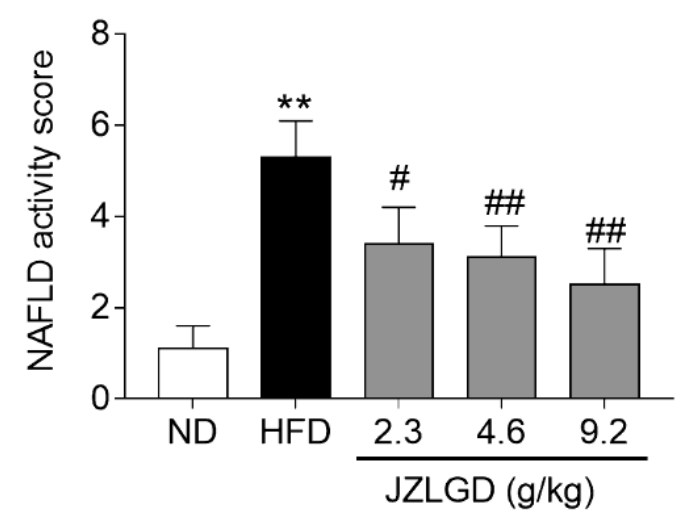

d
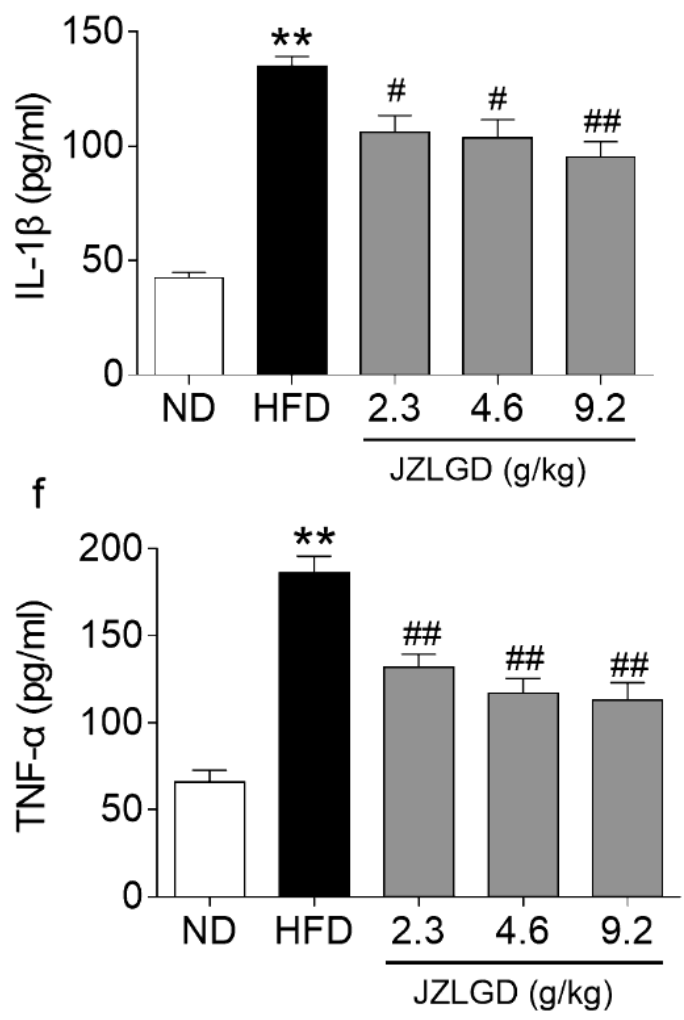

C

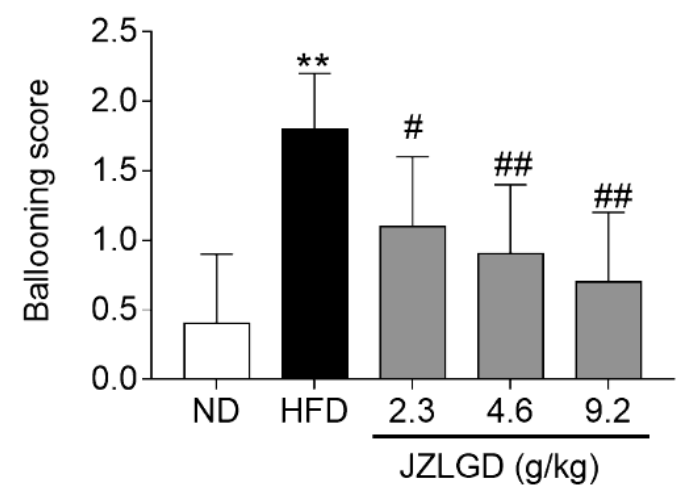

e
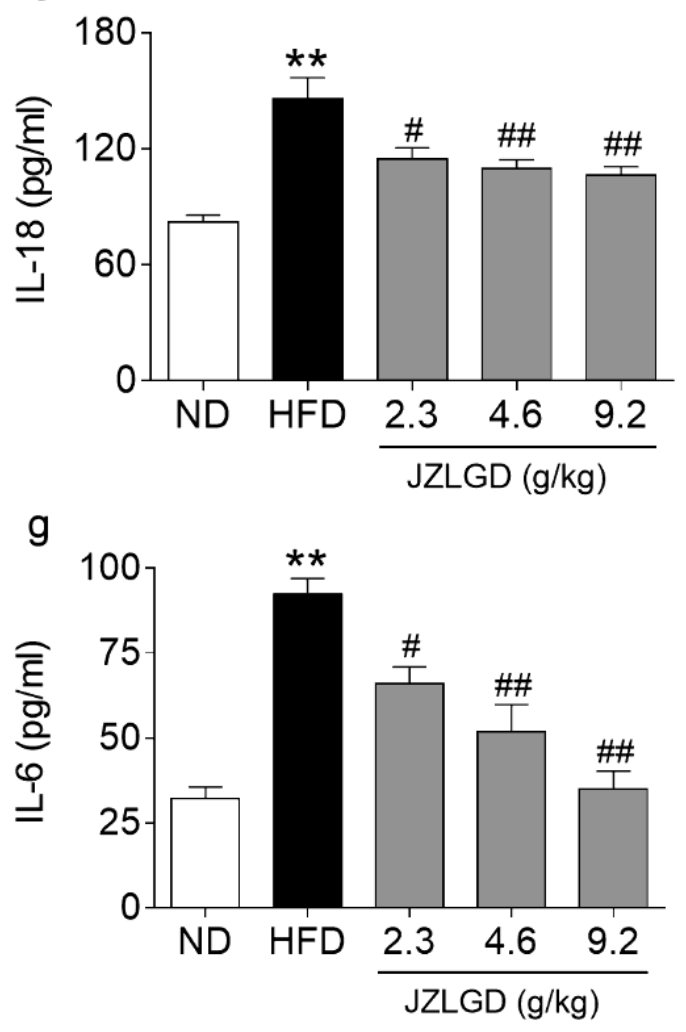

\section{Figure 2}

Effects of JZLGD on liver histology against high fat diet and hepatic inflammatory-related parameters. a H\&E stained sections of liver tissue $(\times 200)$ b The NAFLD activity score c Ballooning degeneration score $d$ Quantitative analysis of serum IL-1 $\beta$ content e Quantitative analysis of serum IL-18 content $f$ Quantitative analysis of serum tumor necrosis factor a(TNF-a) content g Quantitative analysis of serum IL-6 content. Data are expressed as the mean \pm SD $(n=10) ;{ }^{* \star} p<0.01$ vs. control; $\# p<0.05, \# \# p<0.01$ vs. model 
group. JZLGD, Jiangzhi Ligan Decoction; H\&E, hematoxylin and eosin; NAFLD, nonalcoholic fatty liver disease; SD, standard deviation

a

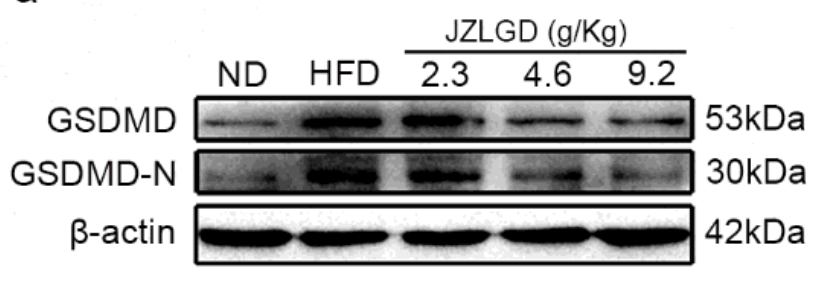

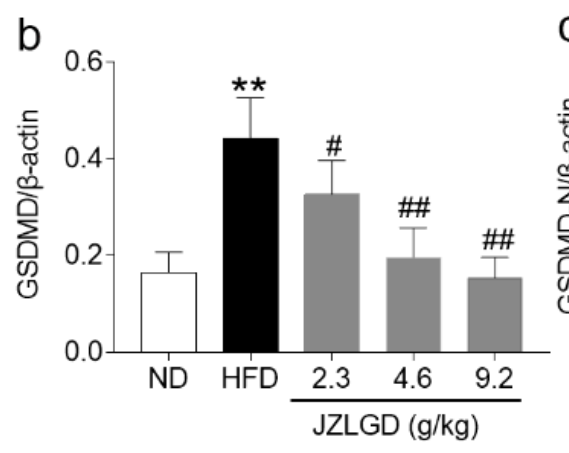

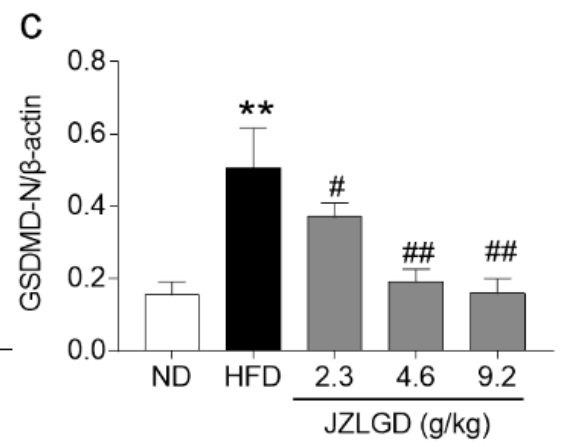

\section{Figure 3}

Effects of JZLGD on the expression of protein GSDMD and GSDMD-N in the liver. a Representative Western blotting results of GSDMD and GSDMD-N; $b$ Densitometric analyses of the band intensity ratios for GSDMD/ $\beta$-actin; $c$ Densitometric analyses of the band intensity ratios for GSDMD-N/ $\beta$-actin. The protein levels are expressed relative to $\beta$-actin. Data are expressed as the mean $\pm S D(n=10) ; * \star p<0.01$ vs. control; $\# p<0.05, \# \# p<0.01$ vs. model group. JZLGD, Jiangzhi Ligan Decoction; SD, standard deviation 
a
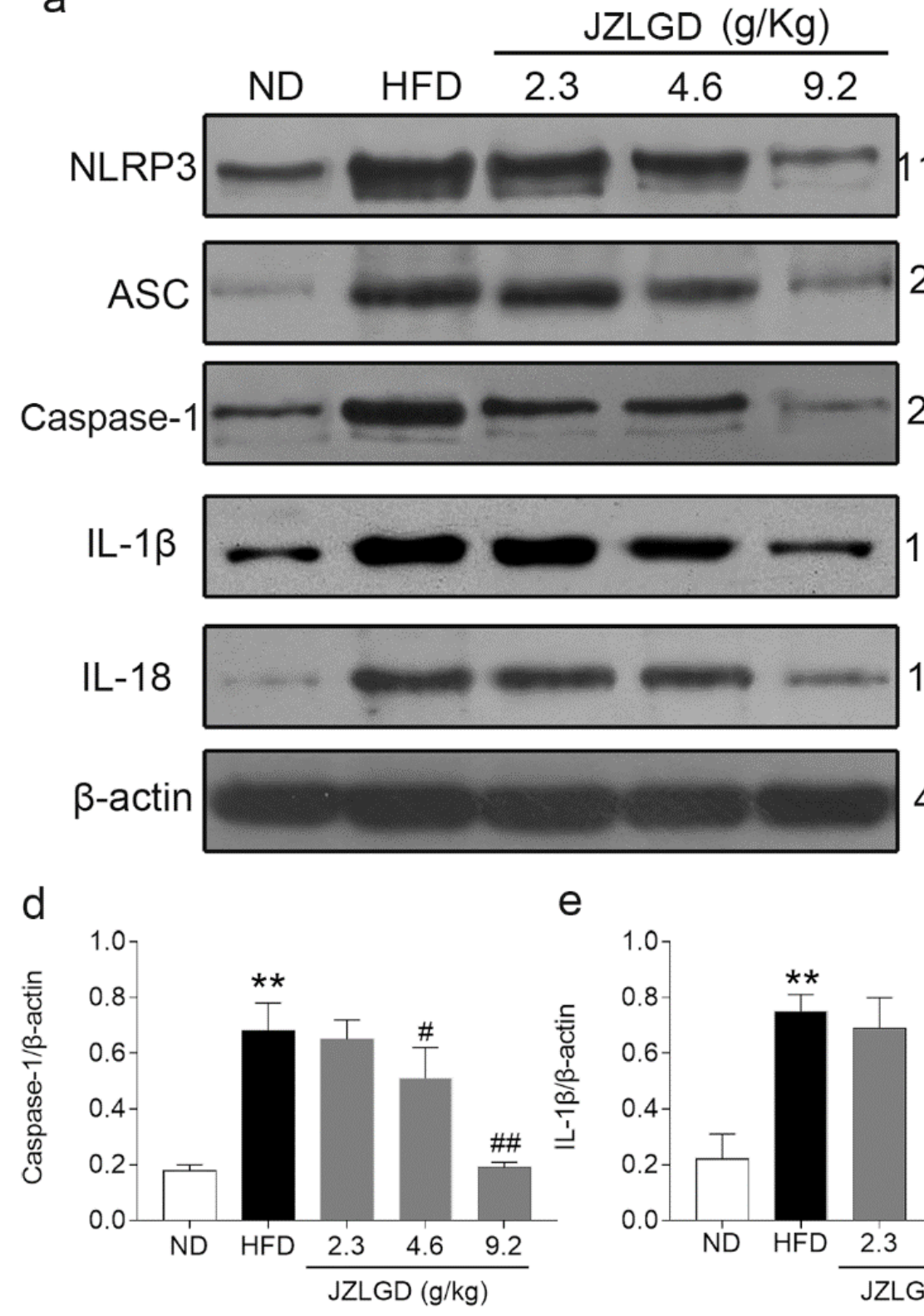

e

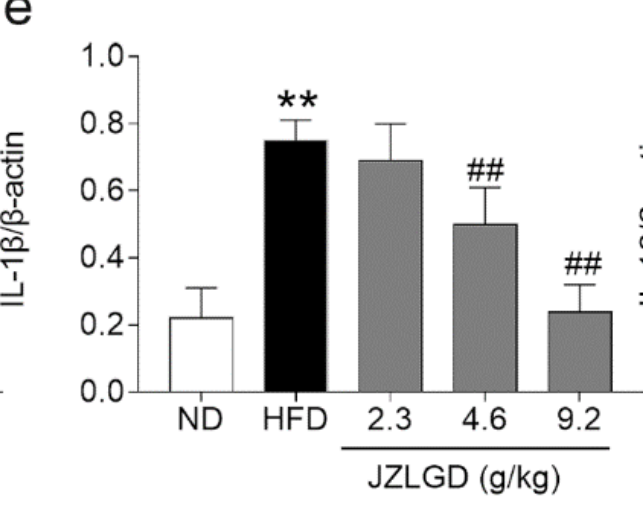

b

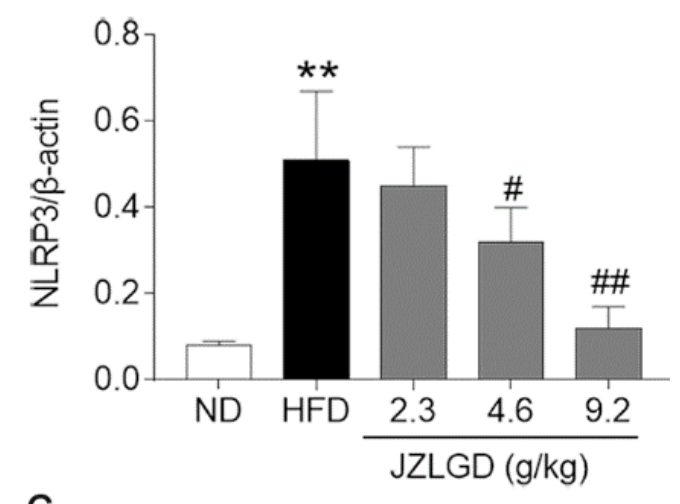

C

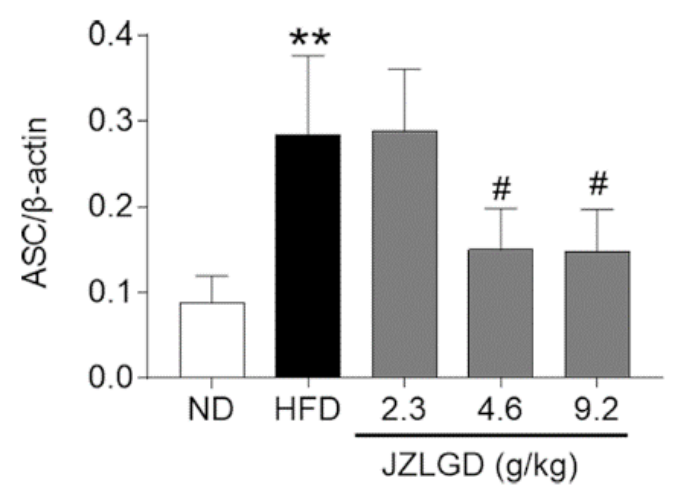

f

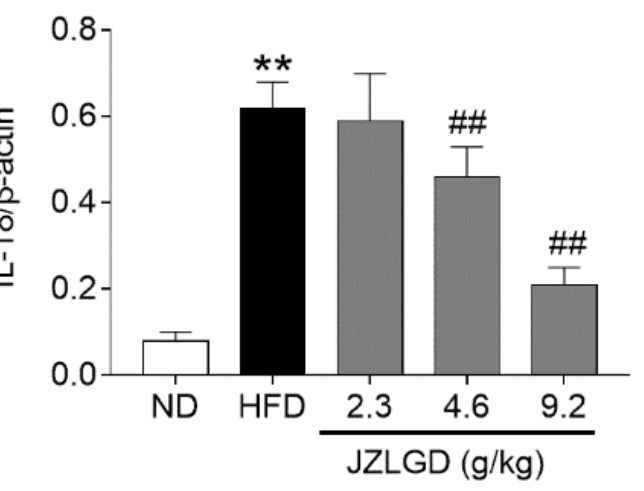

\section{Figure 4}

Effects of JZLGD on the expression of inflammasomes and pro-imflammtory factors on canonical pyroptosis signaling. a Representative Western blotting results of NLRP3, ASC, Caspase-1, IL-1 $\beta$ and IL-18 in the liver; $b$ Densitometric analyses of the band intensity ratios for NLRP3/ $\beta$-actin; $c$ Densitometric analyses of the band intensity ratios for ASC/ $\beta$-actin; $d$ Densitometric analyses of the band intensity ratios for Caspase-1/ $\beta$-actin; e Densitometric analyses of the band intensity ratios for IL-1 $\beta / \beta$-actin; $f$ Densitometric analyses of the band intensity ratios for IL-18/ $\beta$-actin. The protein levels are expressed relative to $\beta$-actin. Data are expressed as the mean $\pm S D(n=10)$; ${ }^{\star \star} p<0.01 \mathrm{vs}$. control; \#p $<0.05$, \#\#p< 0.01 vs. model group. JZLGD, Jiangzhi Ligan Decoction; SD, standard deviation 
a

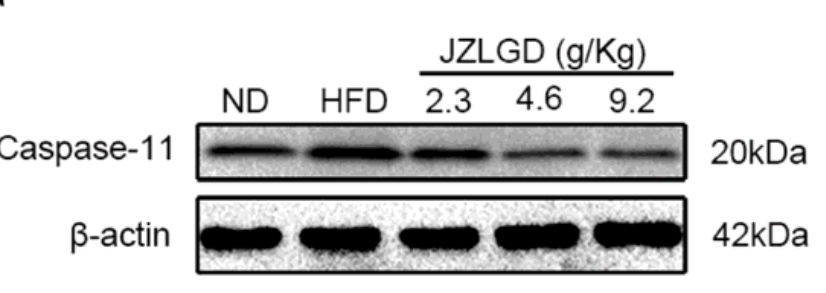

$\mathrm{b}$

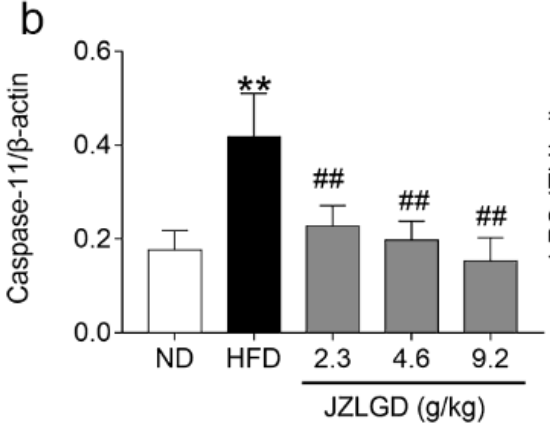

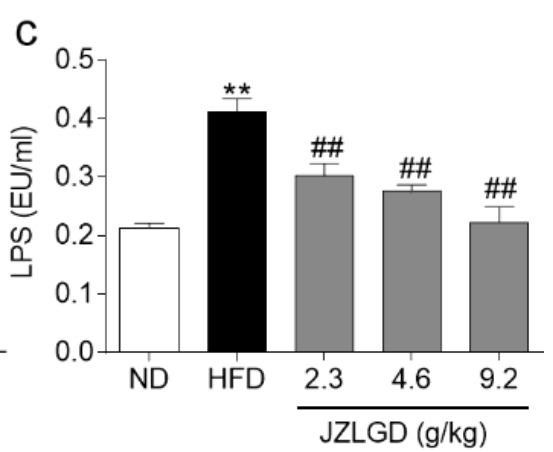

\section{Figure 5}

Effects of JZLGD on the levels of caspase-11 in the liver and LPS in serum. a Representative Western blotting results of Caspase- 11 in the liver , $\beta$-actin was used as internal control; $b$ Densitometric analyses of the band intensity ratios for Caspase-11/ $\beta$-actin; $c$ The level of LPS in serum of NAFLD model rats. Data are expressed as the mean $\pm \operatorname{SD}(n=5) ; * \star p<0.01$ vs. control; \#\#p<0.01 vs. model group. JZLGD, Jiangzhi Ligan Decoction; LPS, lipopolysaccharide; NAFLD, nonalcoholic fatty liver disease; SD, standard deviation

\section{Supplementary Files}

This is a list of supplementary files associated with this preprint. Click to download.

- Graphicalabstract.tif 